\title{
Coconut Oil and its Constituents as a Treatment for Alzheimer's Dementia
}

\author{
Rodney P. Guttmann ${ }^{1}$, Peyton L. Sims ${ }^{1}$, Catherine R. Churchill', Caitlyn R. Waters ${ }^{1}$, \\ Bailey M. Berry ${ }^{1}$ and Jacquelyn J. Wells ${ }^{1}$
}

${ }^{1}$ Department of Biology, Hal Marcus College Department of Science and Engineering, University of West Florida, Pensacola, FL, USA

DOI: $10.47611 /$ jsr.v9i1.1046

\section{ABSTRACT}

In recent years, there has been an increased number of studies focusing on the properties of coconut oil as a therapeutic supplement for patients with Alzheimer's disease. Primarily, benefits of coconut oil are attributed to the presence of medium-chain triglycerides, lauric acid, or ketone bodies found in the oil. Research of these constituents within coconut oil has been shown to cause the onset of cellular processes such as autophagy, ketone body regulation, and the reduction of oxidative stress, among other nonspecific pathways. A discussion of the potential for coconut oil within the context of these theoretical mechanisms to impact Alzheimer's disease is provided, suggesting that the validity of coconut oil claims should be viewed with skepticism.

\section{Introduction}

Alzheimer's disease (AD) is a type of neurodegenerative dementia that affects 5.5 million people in the United States alone. Cognitive symptoms of AD include agnosia, chronic memory loss, visual/spatial impairment, and disorientation. Behavioral symptoms include irritability, word repetition, and personality changes. The gradual destruction of synapses and dendrites are also indicative of AD development [1].

While therapies are available to improve the quality of life in patients with AD and their caregivers, there is currently no treatment or cure for the disease. In the absence of such FDA-approved therapeutics and the failures of recent clinical trials, there remains an attraction to nutriceuticals and other drugs that may have anecdotal evidence and easy access for patients. Coconut oil (CO) is undoubtedly one of the compounds that have reported anecdotal effects and are widely available. For example, claims have been made that propose $\mathrm{CO}$ as a treatment for $\beta$-amyloid plaques [2] and both components found in $\mathrm{CO}$ and metabolites including lauric acid (LA), ketone bodies (KBs), and medium-chain triglyceride (MCTs) have a potential mechanism of action relevant to AD, mainly through KBs [2-5].

\section{MCTs and MCFAs}

$\mathrm{CO}$ is not a single compound but is composed of medium-chain triglycerides (MCTs), which contain medium-chain 
fatty acids (MCFAs) [6]. CO typically contains high percentages of these compounds, with about $100 \%$ of the constituents being MCFAs and approximately $65 \%$ being MCTs [7]. As the primary source of energy of the brain, the metabolism of glucose is the integral piece to regular function [8]. Despite the role of glucose, crude MCTs have been hypothesized to provide an alternative source of energy to improve cognitive function via the breakdown of KBs [9]. However, the extent of this improvement appears to vary among many patients and may not be effective for all individuals treated [10]. The severity and progression of $A D$ is a variable in the success rates of MCT for treatment. Since the fatty acids in MCTs do not effectively cross the blood-brain barrier (BBB), they typically do not serve as a reservoir of energy without further processing [5]. Because of their poor BBB penetration, $\beta$-amyloid plaques and neurofibrillary tangles cannot be significantly affected by fatty acids alone. Thus, the products of MCFAs metabolism, such as $\mathrm{KBs}$, are the more likely link to the use of $\mathrm{CO}$ as AD-related therapy [5].

MCTs supplements have been commercially available both over the counter and by prescription in the United States and Europe [11]. An example of one such product is Axona, a "prescription beverage," consists of MCTs and is proposed to improve cognition through its metabolism to $\beta$-hydroxybutyrate $(\beta-\mathrm{HB})$, a KB [4]. Trials attest that manufactured MCTs alone is not a suitable treatment for $\mathrm{AD}$; in a double-blind study conducted by the manufacturers of Axona, it was determined that the beverage is only useful in APOE E4-negative patients for durations shorter than 90 days [4].

\section{Ketone Bodies}

A mechanism investigated in $\mathrm{CO}$ supplementation is the shift from glucose consumption to the usage of KBs, which serves as an alternate source of energy for brain tissues [12,13]. For example, the two main KBS that are produced during ketone metabolism is acetoacetate (AcAc) and $\beta$-HB $[5,11]$. These compounds, which can be derived from MCTs in $\mathrm{CO}$, are transported into neurons through plasma membrane transporters separate from those utilized by glucose $[3,14]$ and can be used in the Krebs cycle to produce ATP. In AD patients, glucose hypometabolism can produce a glucose deficit of approximately $20-25 \%$ [3]. This deficit can be balanced by increased ketone uptake, and ketone uptake is not significantly different between $\mathrm{AD}$ and healthy, age-matched controls [3].

The usage of KBs has some evidence in increasing cognition in $\mathrm{AD}[11,12,15]$. Gammahydroxybutyrate, and an isomer of $\beta$ - $\mathrm{HB}$, has been found to provide energy and increase antioxidants within the body and thus hypothesized to have potential use in $\mathrm{AD}$ [16]. It was also found that KBs increased motor neuron counts in transgenic mice, although the cognitive performance was not improved [17].

\section{Lauric Acid}

Lauric acid (LA) has been implicated as one of the active compounds within CO, and it constitutes about $50 \%$ of the fatty acids found in CO. LA is absorbed at a slower rate than other long-chain fatty acids but is metabolized similarly. As a significant constituent of $\mathrm{CO}$, the activity of LA directly may be an essential aspect of the actions of CO.

In a study conducted by Nafar and colleagues [2], rats treated with LA were significantly more protected against A $\beta$ toxicity compared to $\mathrm{CO}$ alone. In this study, it was also determined that treatment with $\mathrm{CO}$ and LA increased KBs in treated rats, which, while complicating direct action of LA, further implicates CO metabolism to KBs as a possible 
mechanism of action [2]. Despite the interest in LA, there is currently not sufficient research conducted on the properties of LA to determine its effectiveness as an AD treatment. Currently available studies of the effects of LA on AD are promising but are preliminary and require further investigation before conclusions are drawn; in a study conducted by Harding and colleagues [18], lauric acid was shown to have the ability to cross the BBB and therefore has the potential to induce ketogenesis.

\section{$\mathrm{CO}$ effects not directly associated with specific components of metabolites}

$\mathrm{CO}$ appears to reduce the secretion of A $\beta$ peptides. N2a/APP695 cells were incubated with increasing concentrations of $\mathrm{CO}$ or olive oil as a control. It was found that $0.1 \% \mathrm{CO}$ resulted in a significant reduction in $\mathrm{A} \beta$ peptides, compared to the olive oil. This inhibitory effect altered both APP expression and A $\beta$ peptide production by reducing ARF1 expression at both the protein and mRNA level [19]. ARF1 is a transport protein critical for the secretory pathway to transport APP to the cell surface. The expression of APP on the cell's surface determines the secretion of A $\beta$ peptides. APP is synthesized in the endoplasmic reticulum and is then transported through the trans-Golgi network to the cell surface. In the trans-Golgi network system, ARF1 is an important transport protein because it regulates the construction of a variety of complexes of budding vesicles on the secretory pathways within the Golgi [19]. APP maturation is thus dependent on the trans-Golgi network and ARF1 to transport it to the cell surface.

\section{Discussion}

While $\mathrm{CO}$, likely through $\mathrm{KBs}$, may positively influence $\mathrm{AD}$-related molecular pathways, $\mathrm{CO}$ also has negative effects. While KB's role in lowering total cholesterol and low-density lipoprotein cholesterol has been demonstrated and $\mathrm{KBs}$ in $\mathrm{CO}$ can reduce systolic blood pressure and ameliorate insulin resistance [10], the consumption of large amounts of $\mathrm{CO}$ can be a progenitor in cardiovascular diseases just as in other saturated fats [20]. The dangers of $\mathrm{CO}$ also have many nutritionists suggesting that $\mathrm{CO}$ consumption should be limited, if not wholly avoided [20]. $\mathrm{CO}$ is a complex mixture that has some evidence of modulation of molecular pathways relevant to AD such as energy metabolism, redox state and $\mathrm{A} \beta$ production. The clinical impact of using $\mathrm{CO}$ in therapeutic doses remains questionable, and no clinical trial data demonstrating a positive effect is available. It seems unlikely that any dose of $\mathrm{CO}$ will be therapeutic except in unusual cases in which there is an underlying metabolic issue for which ketone body utilization provides an energy source because glucose is absent. Even in this hypothetical scenario, the benefits of CO would more likely be realized through an altered diet program designed for such a purpose and would need to be implemented as a preventative strategy.

\section{Conclusion}

$\mathrm{CO}$ used to treat or prevent AD is not supported by any sizable peer-reviewed cohort. Still, smaller clinical trials and anecdotal evidence exhibit promising results specifically related to a selection of its active components or metabolism. These components may prove to be a more effective treatment than $\mathrm{CO}$ itself, as the most suitable components of $\mathrm{CO}$ 
can be isolated, purified, and perhaps chemically altered to improve their pharmacology. BHB resulting from MCT metabolism, based on the testing of Axona, does not seem to be a feasible treatment for many patients with AD. Some KBs may prove to be a promising therapy for AD patients, which may be be due to the various mechanisms by which KBs can exert effects. That is because AD appears to have multiple cellular system effects, the treatment will need to mediate a number of cell defects effectively. The dysregulation of glucose, plaque formation, and oxidative stress may require simultaneous modulation for which KB may be useful. Indeed, clinical evaluations of KBs are encouraging when considering both in vitro and in vivo studies. However, more data is needed to determine the longterm effectiveness of ketones in patients with dementia. In addition to this, initial research on the effects of LA presents it as another possible mechanism by which $\mathrm{CO}$ functions, but further testing is needed. Figure 1 represents a summary of $\mathrm{CO}$ potential in AD including a breakdown and general overview of the effectiveness of individual components in $\mathrm{CO}$ in the treatment of $\mathrm{AD}$.

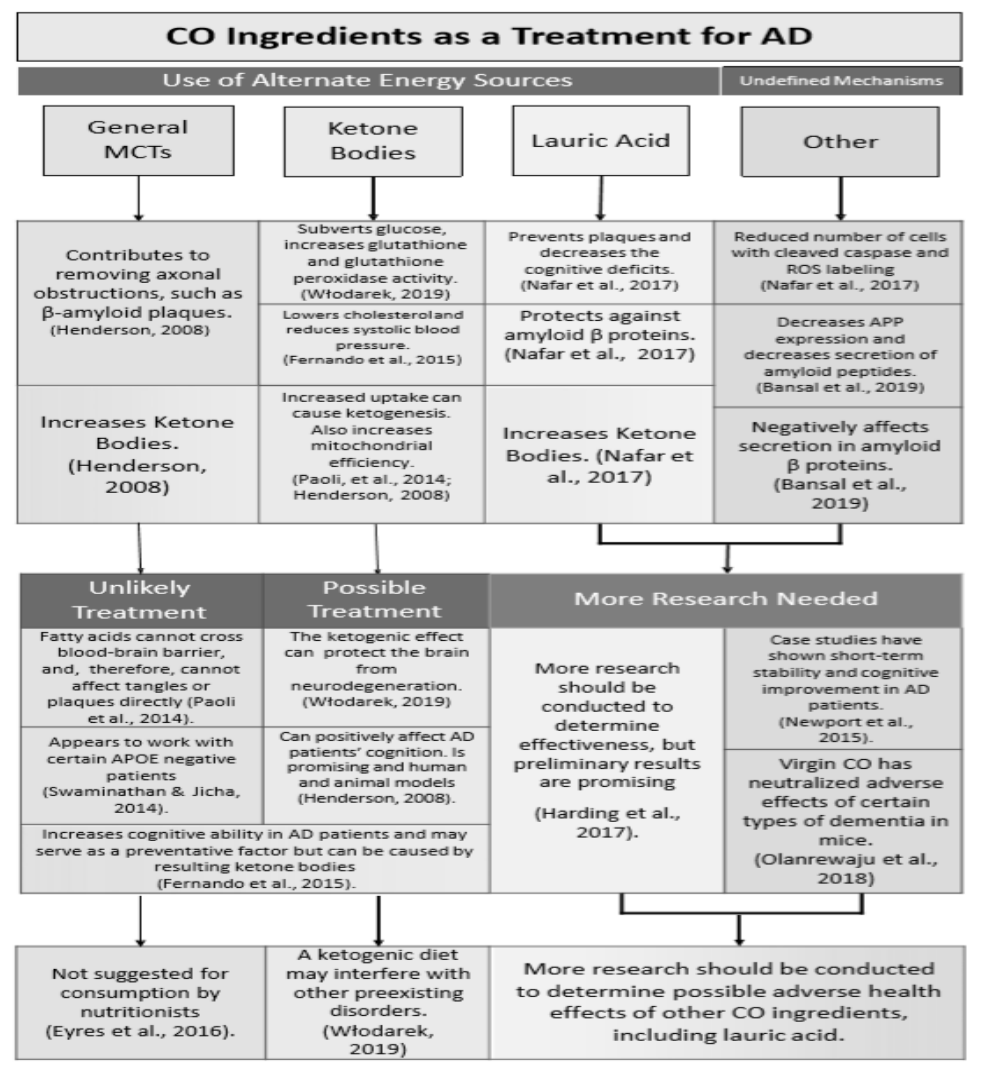

Figure 1. $\mathrm{CO}$ and its ingredients as a treatment for AD.

Currently, CO appears to be an unlikely treatment in itself, but the constituents of CO have therapeutic potential. KBs in particular have shown promise in increasing cognitive function in both human and animal models. Despite this, the ketogenic diet, can prove difficult for AD patients to follow. Furthermore, LA and other constituents of CO have shown preliminary results that demonstrate patients exhibiting a stability in condition. 


\section{Acknowledgments}

The authors would like to thank the University of West Florida Biology Department for the extensive amount of resources provided to us in the process of research.

\section{Author Contributions}

All authors certify that they have participated sufficiently in the work to take public responsibility for the content, including participation in the research, analysis, writing, or revision of the manuscript.

\section{Declaration of Interests}

No referenced author received any gain, financial or otherwise, for opinions given or expressed in present representation. No auxiliary motivation towards any particular perceived bias was made.

\section{References}

1. Huang, W.J., X. Zhang, and W.W. Chen, Role of oxidative stress in Alzheimer's disease. Biomed Rep, 2016. 4(5): p. 519-522.

2. Nafar, F., J.P. Clarke, and K.M. Mearow, Coconut oil protects cortical neurons from amyloid beta toxicity by enhancing signaling of cell survival pathways. Neurochem Int, 2017. 105: p. 64-79.

3. Newport, M.T., et al., A new way to produce hyperketonemia: use of ketone ester in a case of Alzheimer's disease. Alzheimers Dement, 2015. 11(1): p. 99-103.

4. Thaipisuttikul, P. and J.E. Galvin, Use of medical foods and nutritional approaches in the treatment of Alzheimer's disease. Clin Pract (Lond), 2012. 9(2): p. 199-209.

5. Paoli, A., Ketogenic diet for obesity: friend or foe? Int J Environ Res Public Health, 2014. 11(2): p. 2092107.

6. Wang, Y., et al., Medium Chain Triglycerides enhances exercise endurance through the increased mitochondrial biogenesis and metabolism. PLoS One, 2018. 13(2): p. e0191182.

7. DebMandal, M. and S. Mandal, Coconut (Cocos nucifera L.: Arecaceae): in health promotion and disease prevention. Asian Pac J Trop Med, 2011. 4(3): p. 241-7. 
8. Mergenthaler, P., et al., Sugar for the brain: the role of glucose in physiological and pathological brain function. Trends Neurosci, 2013. 36(10): p. 587-97.

9. Augustin, K., et al., Mechanisms of action for the medium-chain triglyceride ketogenic diet in neurological and metabolic disorders. Lancet Neurol, 2018. 17(1): p. 84-93.

10. Fernando, W.M., et al., The role of dietary coconut for the prevention and treatment of Alzheimer's disease: potential mechanisms of action. Br J Nutr, 2015. 114(1): p. 1-14.

11. Cunnane, S.C., et al., Can Ketones Help Rescue Brain Fuel Supply in Later Life? Implications for Cognitive Health during Aging and the Treatment of Alzheimer's Disease. Front Mol Neurosci, 2016. 9: p. 53.

12. Cunnane, S.C., et al., Can ketones compensate for deteriorating brain glucose uptake during aging? Implications for the risk and treatment of Alzheimer's disease. Ann N Y Acad Sci, 2016. 1367(1): p. 12-20.

13. Włodarek, D., Role of Ketogenic Diets in Neurodegenerative Diseases (Alzheimer's Disease and Parkinson's Disease). Nutrients, 2019. 11(1).

14. Vandenberghe, C., et al., Tricaprylin Alone Increases Plasma Ketone Response More Than Coconut Oil or Other Medium-Chain Triglycerides: An Acute Crossover Study in Healthy Adults. Curr Dev Nutr, 2017. 1(4): p. e000257.

15. Gandotra, S., J. Kour, and A. Van der Waag, Efficacy of adjunctive extra virgin coconut oil use in moderate to severe Alzheimer's disease. International Journal of School and Cognitive Psychology, 2014. 1(2).

16. Mamelak, M., Sporadic Alzheimer's disease: the starving brain. J Alzheimers Dis, 2012. 31(3): p. 459-74.

17. Henderson, S.T., Ketone bodies as a therapeutic for Alzheimer's disease. Neurotherapeutics, 2008. 5(3): p. $470-80$.

18. Harding, A., et al., Exploring the Association between Alzheimer's Disease, Oral Health, Microbial Endocrinology and Nutrition. Front Aging Neurosci, 2017. 9: p. 398.

19. Bansal, A., et al., Coconut oil decreases expression of amyloid precursor protein (APP) and secretion of amyloid peptides through inhibition of ADP-ribosylation factor 1 (ARF1). Brain Res, 2019. 1704: p. 78-84.

20. Eyres, L., et al., Coconut oil consumption and cardiovascular risk factors in humans. Nutr Rev, 2016. 74(4): p. 267-80. 